\title{
REVISTAMATËRIA
}

\section{Avaliação do concreto reforçado com baixos teores de fibras para fins estruturais segundo a norma EN 14651-2007}

\author{
Assessment of concrete reinforced with \\ low content of fibres for structural \\ purposes according to standard \\ EN 14651-2007
}

Renan Pícolo Salvador ${ }^{1}$, Juliana Ferreira Fernandes ${ }^{1}$, Antonio Domingues de Figueiredo ${ }^{1}$

\footnotetext{
${ }^{1}$ Departamento de Engenharia de Construção Civil da Escola Politécnica da Universidade de São Paulo, São Paulo, SP. e-mail: renan.salvador@usp.br; julianaffernandes@gmail.com; antonio.figueiredo@usp.br
}

\section{RESUMO}

Dentre os diversos métodos de avaliação do comportamento mecânico de concreto reforçado com fibras (CRF), o ensaio proposto pela norma EN 14651-2007 é um dos mais promissores. Isto ocorre porque o mesmo é base da nova proposta do fib Model Code para o dimensionamento de estruturas utilizando o CRF. Dado que não há normas técnicas brasileiras abordando esse tipo de ensaio, é necessário empregar este método de ensaio na avaliação do CRF. Para tal, um estudo experimental foi desenvolvido focando o uso deste método de ensaio para a qualificação e dosagem de concretos reforçados com baixos teores de fibras de aço e de macrofibras poliméricas. Foram realizados ensaios de flexão de corpos-de-prova prismáticos com entalhe na face inferior, cuja função é induzir o posicionamento da fissura. Foram estudados três tipos de fibra, duas compostas de polipropileno (nas dosagens de 3,0, 4,5 e 6,0 kg/ $\mathrm{m}^{3}$ - correspondentes a 0,33, 0,50 e 0,66\% em volume, respectivamente) e uma de aço (nas dosagens de 15, 25 e $35 \mathrm{~kg} / \mathrm{m}^{3}$ - correspondentes a 0,19, 0,32 e 0,45\% em volume, respectivamente) em uma matriz de concreto de resistência à compressão média de 35MPa. Verificou-se grande estabilidade nos resultados dos ensaios, com baixa dispersão nos resultados. Foi comprovado que é possível dosar as fibras de modo a atender diferentes níveis de requisitos estabelecidos no fib Model Code para o CRF destinado a aplicações estruturais quando são considerados os valores médios dos resultados de ensaio. Esta situação pode não se repetir quando os resultados característicos são considerados.

Palavras-chave: concreto reforçado com fibras, estruturas de concreto, método de ensaio, resistência residual.

\footnotetext{
ABSTRACT

There is a broad range of tests available for evaluating the mechanical behavior of fiber-reinforced concrete (FRC). The one proposed by the standard EN 14651-2007 is one of the most promising. This is due to the fact that this test method was chosen as reference in the new fib Model Code for structural design using the FRC. Since there is no standard published in Brazil regarding that kind of test, there is a need of use this test method in the evaluation of CRF. In that sense, an experimental program was carried out aiming to proceed a mix-design analysis of FRC and verify if concretes reinforced with low content of synthetic macrofibers and steel fibers could be in conformity to requirements for structural application according to the fib Model Code. Tests were performed using notched prismatic specimens, in which the formation of the crack is more stable. Three types of fiber have been evaluated, two of them were composed of polypropylene, used in the dosages of $3.0,4.5$ and $6.0 \mathrm{~kg} / \mathrm{m}^{3}(0.33,0.50$ and 0.66 in volume percentage) and the third one was composed of steel, used in the dosages of 15,25 and $35 \mathrm{~kg} / \mathrm{m}^{3}(0.19,0.32$ and 0.45 in volume percentage) in one concrete matrix of 35MPa of compressive strength. Great stability and reproducible results were achieved.
} 
It has been proven that it is possible to dose the fibers in order to meet different levels of requirements established in the fib.

Model Code for FRC designated to structural purposes when considering mean values of test results. This situation can not be repeated when the characteristic results are considered in the analysis.

Keywords: fiber-reinforced concrete, concrete structures, test method, residual strength.

\section{INTRODUÇÃO}

O concreto, material estrutural amplamente utilizado em todo o mundo, tem sido assunto de pesquisas contínuas no ramo da construção. Em parte, isso ocorre devido ao fato desse compósito apresentar certas limitações, como a baixa capacidade de deformação e a rápida propagação de fissuras quando submetido a esforços de tração [1,2].

As fibras de aço são utilizadas há bastante tempo, podendo ser consideradas um tipo de reforço tradicional para o concreto. Apesar disso, há ainda uma série de aspectos tecnológicos deste tipo de reforço que ainda precisam de melhor desenvolvimento. Uma evidência desta condição é a carência de normas técnicas publicadas sobre o assunto no Brasil, onde há apenas uma especificação para a fibra como material [3], não havendo ainda normas que regulem o concreto com fibras (CRF). Neste sentido, um dos temas prioritários é a definição de um método padrão de avaliação da tenacidade. Para isso, é importante haver uma coerência entre o método de ensaio e o modelo de dimensionamento da estrutura usando o CRF.

No Brasil, existe uma proposição de diretrizes de projeto feita pela ABECE [4]], a qual é fortemente baseada no modelo proposto pelo código modelo fib [5, 6]. Segundo o novo código modelo fib, o ensaio básico de caracterização do CRF é o de flexão de prismas com entalhe estabelecido na norma EN 14651-2007 [7]. Como outros ensaios tradicionais [8-10], o ensaio EN 14651 é baseado na flexão de prismas e na determinação da resistência residual do CRF. No entanto, estes ensaios apresentam distintos níveis de dificuldade de aplicação. O ensaio da JSCE [10] é o de execução mais simples e mais amplamente aplicado no Brasil. Já o ensaio ASTM C1609 [8] segue um procedimento similar, mas exige o uso de equipamentos mais sofisticados, dotados de sistema fechado de controle de deslocamento. O sistema aberto corresponde àquele em que a taxa de deformação imposta ao corpo-de-prova é determinada pelo deslocamento da prensa enquanto o sistema fechado controla a velocidade de deformação pelo próprio sistema de medida da mesma [11]. Ou seja, no sistema fechado a velocidade do ensaio é definida pela velocidade constante de deformação medida pelo transdutor acoplado ao corpo-de-prova. Esta maior exigência garante um nível maior de precisão na avaliação como já foi demonstrado em estudo anterior [11]. No entanto, a exigência da norma EN 14651-2007 [7] de utilização de entalhe no corpo-de-prova torna o método ainda mais trabalhoso. Talvez por isso, a avaliação do comportamento mecânico e estrutural do CRF com distintos tipos de fibras já foi objeto de vários estudos internacionais [2, 12-15] e brasileiros [11, 16-21], sem a utilização deste método específico. Assim, há a necessidade de utilização deste método para a caracterização do comportamento mecânico e dosagem do CRF de modo a atender aos critérios estabelecidos no fib Model Code para aplicações estruturais do concreto reforçado com distintos tipos de fibras em baixos teores[22].

Com o intuito de contribuir para a minimização dessas limitações tecnológicas, foi realizado um estudo experimental utilizando ensaios de flexão de corpos-de-prova com entalhe, dado que é uma alternativa interessante pelo vínculo que o mesmo apresenta com as novas propostas de modelo de dimensionamento de estruturas de CRF. Além disso, é um ensaio que tem o potencial de reduzir a variabilidade do resultado experimental. Isto ocorre porque a formação da fissura ocorre na região do plano de entalhe, minimizando a dissipação de energia no corpo-de-prova. Portanto, a absorção de energia é diretamente atribuída à fratura ao longo desse plano [5, 23]. Este estudo se encontra detalhado a seguir.

\section{MATERIAIS E MÉTODOS}

Para avaliar o comportamento mecânico do concreto reforçado com fibras, o método utilizado foi o proposto pela norma EN 14651 (2007) [7]. Este procedimento experimental prescreve ensaios de flexão de corpos-deprova prismáticos, cujas dimensões são $150 \times 150 \times 550 \mathrm{~mm}^{3}$, com vão de ensaio de $500 \mathrm{~mm}$, ideais para utilização de agregados graúdos de diâmetro máximo de $32 \mathrm{~mm}$. Entretanto, devido à utilização de agregados graúdos de diâmetro máximo de $19 \mathrm{~mm}$, os corpos-de-prova utilizados tinham as dimensões de 150x150x500 $\mathrm{mm}^{3}$ e foram ensaiados com vão de $450 \mathrm{~mm}$.

Os corpos-de-prova possuíam um entalhe, de $5 \mathrm{~mm}$ de largura por $25 \mathrm{~mm}$ de altura, centralizado em sua face inferior, que ficava apoiada nos cutelos da base da prensa. A função do entalhe é induzir o posicionamento da fissura. A carga foi aplicada por apenas um cutelo superior, centralizado em relação ao vão de 
ensaio. O deslocamento vertical do corpo-de-prova foi a variável de controle do ensaio. O esquema da execução do ensaio está mostrado na Figura 1.

Foram utilizadas duas velocidades de aplicação de carga: no primeiro trecho, correspondente ao intervalo de abertura de fissura entre 0 e $0,10 \mathrm{~mm}$, a velocidade de deslocamento vertical foi de $0,05 \mathrm{~mm} / \mathrm{min}$. No segundo, entre 0,10 e $4,0 \mathrm{~mm}$, a velocidade foi de $0,20 \mathrm{~mm} / \mathrm{min}$. Foi adotada essa menor velocidade no trecho inicial, pois esta é a etapa crítica da realização dos ensaios, onde a carga é transferida da matriz para as fibras. Esse intervalo de deslocamento está associado ao trecho elástico $(0$ a $0,05 \mathrm{~mm}$ de deslocamento vertical, aproximadamente) e ao início do comportamento elasto-plástico. No segundo trecho, correspondente ao comportamento pós-fissuração, foi adotado maior velocidade de ensaio, pois não é esperado nenhum tipo de instabilidade nesta etapa.

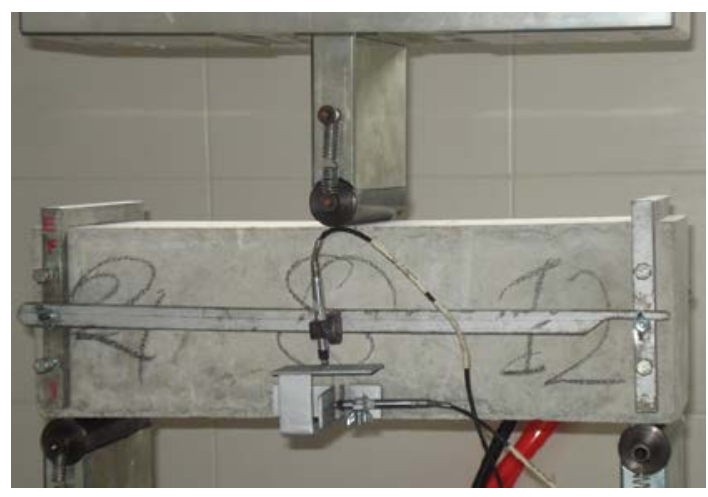

(a)

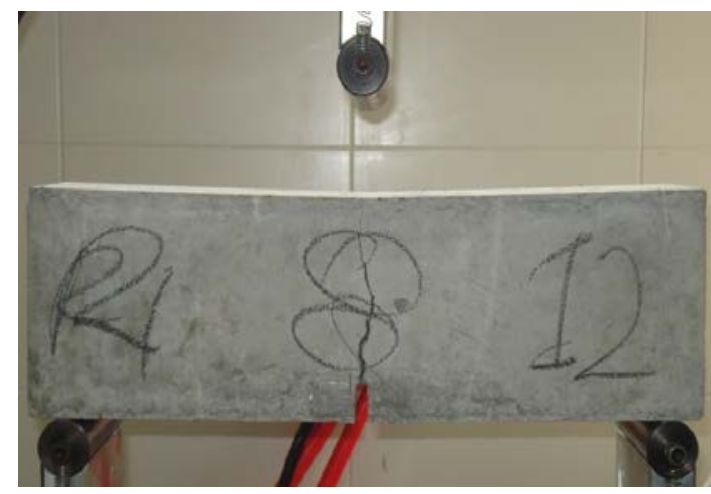

(b)

Figura 1: Esquema de realização do ensaio: preparação do corpo-de-prova (a) e corpo-de-prova rompido (b).

Foram avaliadas três fibras para reforço de concreto, duas poliméricas e uma de aço. Foi utilizada uma matriz (resistência média à compressão de $35 \mathrm{MPa})$, com dosagens de $3,0,4,5$ e $6,0 \mathrm{~kg} / \mathrm{m}^{3}(0,33,0,50$ e $0,66 \%$ em volume, respectivamente) para as macrofibras sintéticas e de 15,25 e $35 \mathrm{~kg} / \mathrm{m}^{3}(0,19,0,32$ e $0,45 \%$ em volume, respectivamente) para a fibra de aço. O procedimento de moldagem dos corpos-de-prova foi realizado segundo o método JSCE-SF2 [24]. Para cada traço, foram moldados quatro corpos-de-prova prismáticos para ensaio de flexão e três cilíndricos, de dimensões $15 \times 30 \mathrm{~cm}$ (diâmetro x altura) para ensaio de compressão, segundo ABNT 5739 (2007) [25]. No caso de haver problemas no ensaio de flexão, de acordo como prescrito na norma, ao menos três corpos de prova foram utilizados para a avaliação do material.

As Tabelas 1 e 2 apresentam os consumos, a composição e as características do traço de concreto utilizado no estudo. As fibras utilizadas nos ensaios estão representadas na Figura 2 onde se pode notar que as macrofibras de PP são fornecidas de duas maneiras distintas. A macrofibra PP A é produzida em feixes compostos por vários filamentos que são enrolados como pequenas cordas. Já a macrofibra PP B é fornecida totalmente solta. A finalidade de produção da macrofibra PP A em feixes é reduzir o risco de embolamentos que são mais frequentes quando a fibra possui maior fator de forma [1]. Além disso, essa fibra é fornecida ao mercado composta por dois tipos de fibras: a macrofibra propriamente dita de cor escura e com maior resistência e módulo de elasticidade e uma fibra de polipropileno de baixo módulo e elevado fator de forma transparente. A intenção do produtor é fornecer um sistema híbrido que garanta reforço do concreto endurecido (macrofibra escura) e controle de retração no estado plástico (fibra transparente) [22]. Estas fibras foram utilizadas exatamente como eram fornecidas no mercado. As propriedades das fibras, de acordo com os informativos técnicos dos fabricantes, estão listadas na Tabela 3. O valor especificado para o abatimento é o de referência para a matriz sem a adição das fibras. Neste estudo, optou-se por elevar este valor a $120 \mathrm{~mm}$ e não proceder a correções na dosagem da matriz para garantir o mesmo nível de abatimento com o aumento do teor de fibra. Este abatimento não foi corrigido pelo fato de se utilizar baixos volumes de fibra, o que não proporciona grande prejuízo à compactação quando o material é vibrado [26]. 
Tabela 1: Consumos dos materiais para a produção do concreto utilizado no estudo.

\begin{tabular}{c|c}
\hline Material & Dosagem $\mathbf{( k g / \mathbf { m } ^ { 3 } )}$ \\
\hline Cimento CPV ARI & 330,00 \\
\hline Agregado miúdo & 825,00 \\
\hline Agregado graúdo & 1023,00 \\
\hline Água & 198,00 \\
\hline Aditivo polifuncional & 1,32 \\
\hline
\end{tabular}

Tabela 2: Traço e características do concreto.

\begin{tabular}{c|c}
\hline Traço unitário & $1,00: 2,50: 3,10: 0,60$ \\
\hline Massa específica $/ \mathrm{kg} / \mathrm{m}^{3}$ & 2287 \\
\hline Ar incorporado / \% & 3,2 \\
\hline Abatimento / mm & 120 \\
\hline Teor de argamassa seca / \% & 53,0 \\
\hline $\mathrm{f}_{\mathrm{cm}} / \mathrm{MPa}$ & 35 \\
\hline
\end{tabular}

Tabela 3: Propriedades das fibras utilizadas, de acordo com informativos técnicos dos fabricantes.

\begin{tabular}{c|c|c|c}
\hline Propriedade & PP A & PP B & Aço \\
\hline Material & copolímero de polipropileno virgem & olefina modificada & aço \\
\hline Forma & monofilamento / fibrilado & monofilamento & Tipo A1 \\
\hline Número de filamentos por quilograma & 221000 & 37000 & 4600 \\
\hline Número de filamentos por litro & 201100 & 33300 & 36100 \\
\hline Comprimento / mm & 54 & 54 & 60 \\
\hline Diâmetro equivalente / mm & 0,32 & 0,90 & 0,75 \\
\hline Densidade / g/cm ${ }^{3}$ & 0,91 & 0,91 & 7,85 \\
\hline Módulo de elasticidade / GPa & 5 & 10 & 210 \\
\hline Resistência à tração / MPa & $570-660$ & 640 & 1100
\end{tabular}

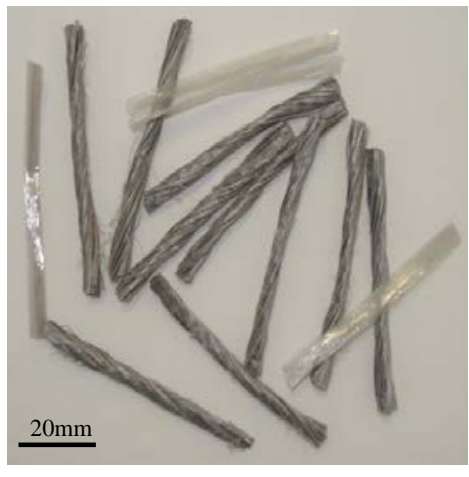

(a)

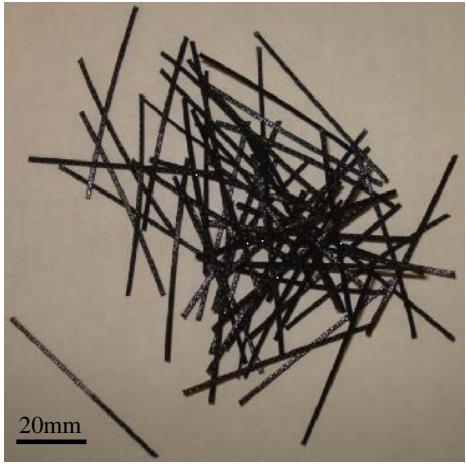

(b)

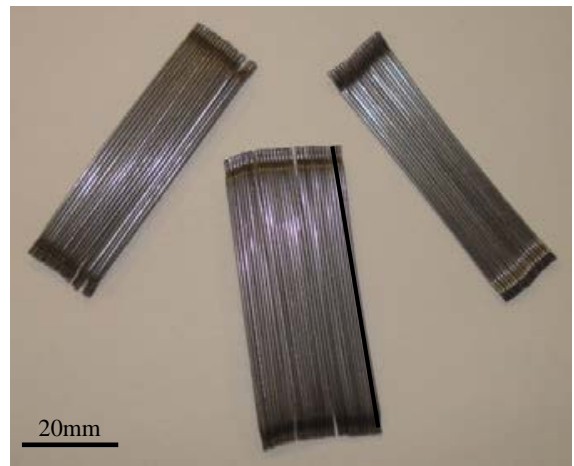

(c) 
Figura 2: Fibras utilizadas neste estudo: macrofibra PP A (a); macrofibra PP B (b) e fibra de aço (c).

Para a análise da curva carga-abertura de fissura segundo a norma EN 14651, são utilizadas duas equações principais. Com a equação 1, é calculada a resistência da matriz correspondente ao limite de proporcionalidade. Já a equação 2 é utilizada para calcular as resistências residuais em 0,50, 1,50, 2,50 e $3,50 \mathrm{~mm}$ de abertura de fissura $\left(\mathrm{f}_{\mathrm{R}, 1}, \mathrm{f}_{\mathrm{R}, 2}, \mathrm{f}_{\mathrm{R}, 3}\right.$ e $\mathrm{f}_{\mathrm{R}, 4}$, respectivamente).

$$
\begin{aligned}
& \mathrm{f}_{\mathrm{L}}=\left(3 \cdot \mathrm{F}_{\mathrm{L}} \cdot \mathrm{l}\right) /\left(2 \cdot b \cdot \mathrm{h}_{\mathrm{sp}}{ }^{2}\right) \\
& \mathrm{f}_{\mathrm{R}, \mathrm{j}}=\left(3 \cdot \mathrm{F}_{\mathrm{j}} \cdot \mathrm{l}\right) /\left(2 \cdot \mathrm{b} \cdot \mathrm{h}_{\mathrm{sp}}{ }^{2}\right)
\end{aligned}
$$

Onde,

$\mathrm{f}_{\mathrm{L}}$ : limite de proporcionalidade, também abreviado por LOP (MPa);

$f_{R, j}$ : resistência residual à flexão correspondente à abertura de fissura $j$, onde $j=0,50,1,5,2,5$ ou 3,5mm $\mathrm{f}_{\mathrm{R}, 1}, \mathrm{f}_{\mathrm{R}, 2}, \mathrm{f}_{\mathrm{R}, 3}$ e $\mathrm{f}_{\mathrm{R}, 4}$, respectivamente (MPa);

$\mathrm{F}_{\mathrm{L}}$ : carga máxima de ensaio no intervalo de abertura de fissura de 0 a $0,50 \mathrm{~mm}(\mathrm{~N})$;

$F_{j}$ : carga correspondente à abertura de fissura $j$, onde $j=0,50,1,5,2,5$ ou $3,5 \mathrm{~mm}-F_{1}, F_{2}, F_{3}$ e $F_{4}$, respectivamente $(\mathrm{N})$;

l: vão de ensaio (mm);

b: largura do corpo-de-prova (mm);

$\mathrm{h}_{\mathrm{sp}}$ : distância entre o topo do entalhe e a face superior do corpo-de-prova (mm).

Para facilitar a classificação do material, seu desempenho no estado limite último $\left(\mathrm{f}_{\mathrm{R}, 3 \mathrm{k}}\right)$ pode ser associado ao do estado limite de serviço $\left(f_{R, 1 k}\right)$ através da relação $f_{R, 3 k} / f_{R, 1 k}$. Segundo o fib Model Code 2010 [6], para garantir o bom desempenho do CRF em elementos estruturais, o mesmo deve apresentar um nível mínimo de ductilidade, o que é verificado pelo atendimento das relações apresentadas nas equações 3 e 4 .

$$
\begin{aligned}
& \mathrm{f}_{\mathrm{R}, 1 \mathrm{k}} / \mathrm{f}_{\mathrm{Lk}} \geq 0,4 \\
& \mathrm{f}_{\mathrm{R}, 3 \mathrm{k}} / \mathrm{f}_{\mathrm{R}, 1 \mathrm{k}} \geq 0,5
\end{aligned}
$$

Onde,

$\mathrm{f}_{\mathrm{Lk}}$ : resistência nominal característica do concreto (MPa);

$\mathrm{f}_{\mathrm{R}, 1 \mathrm{k}}$ : resistência residual característica do concreto para $0,5 \mathrm{~mm}$ de abertura de fissura (MPa);

$\mathrm{f}_{\mathrm{R}, 3 \mathrm{k}}$ : resistência residual característica do concreto para 2,5 mm de abertura de fissura (MPa).

Há também uma classificação do comportamento do CRF quanto à tenacidade prevista pelo fib Model Code baseada na relação $\mathrm{f}_{\mathrm{R} 3 \mathrm{k}} / \mathrm{f}_{\mathrm{R} 1 \mathrm{k}}$. Nessa classificação são estabelecidas categorias de comportamento residual (a, b, c, d, e) considerando que o valor de $f_{R 1 k}$ pode variar de $1 \mathrm{MPa}$ a $8 \mathrm{MPa}$ e a relação $\mathrm{f}_{\mathrm{R} 3 \mathrm{k}} / \mathrm{f}_{\mathrm{R} 1 \mathrm{k}}$ pode se circunscrever nos seguintes intervalos de classificação [5,6]:

- $\quad$ a se $0.5 \leq \mathrm{f}_{\mathrm{R} 3 \mathrm{k}} / \mathrm{f}_{\mathrm{R} 1 \mathrm{k}} \leq 0.7$

- $\quad$ s se $0.7 \leq \mathrm{f}_{\mathrm{R} 3 \mathrm{k}} / \mathrm{f}_{\mathrm{R} 1 \mathrm{k}} \leq 0.9$

- $\quad$ se $0.9 \leq \mathrm{f}_{\mathrm{R} 3 \mathrm{k}} / \mathrm{f}_{\mathrm{R} 1 \mathrm{k}} \leq 1.1$

- $\quad d$ se $1.1 \leq \mathrm{f}_{\mathrm{R} 3 \mathrm{k}} / \mathrm{f}_{\mathrm{R} 1 \mathrm{k}} \leq 1.3$

- $\quad$ e se $1.3 \leq \mathrm{f}_{\mathrm{R} 3 \mathrm{k}} / \mathrm{f}_{\mathrm{R} 1 \mathrm{k}}$

Assim, um CRF que apresente uma resistência residual $\mathrm{f}_{\mathrm{R} 1 \mathrm{k}}$ de $2 \mathrm{MPa}$ e uma relação $\mathrm{f}_{\mathrm{R} 3 \mathrm{k}} / \mathrm{f}_{\mathrm{R} 1 \mathrm{k}}$ de 0,95 , seria classificado como 2c.Note-se que os índices " $k$ ” dos valores apontados nas equações 3 e 4, bem como nas classificações indicadas acima, correspondem aos valores característicos das medidas de resistência residual. Para o presente estudo, considerou-se a condição do valor característico como aquele que garante que 95\% da população estará acima deste valor de referência. Portanto, a determinação dos valores característicos seguiu a formulação convencional apresentada na equação 5. Há orientações para a consideração do valor característico como 70\% do valor médio [27], no entanto, isto implica em uma análise simplista da variabilidade do material. Por isso, julgou-se conveniente a abordagem segundo a estatística básica.

$$
\mathrm{f}_{\mathrm{k}}=\mathrm{f}_{\mathrm{m}}-1,65 . \mathrm{S}_{\mathrm{D}}
$$

Onde, 
$\mathrm{f}_{\mathrm{k}}$ : valor característico;

$\mathrm{f}_{\mathrm{m}}$ : valor médio;

$\mathrm{S}_{\mathrm{D}}$ : desvio padrão da amostra.

\section{RESULTADOS E DISCUSSÃO}

\subsection{Ensaio de compressão}

Os resultados de resistência à compressão estão mostrados na Tabela 4. De acordo com os dados obtidos, verifica-se que houve baixa variação entre os resultados. Com os traços utilizados, a resistência à compressão é praticamente independente do teor de fibra. Tal fato já era esperado e é confirmado por dados presentes na literatura [5, 28-30]. Isto também comprova o fato de não ter havido influência da fibra na condição de compactação do material, mesmo não tendo sido corrigido o nível de abatimento da mistura. A resistência à compressão média entre todos os ensaios foi de 33,9 MPa, com coeficiente de variação de 3,7\%. Embora o valor tenha ficado ligeiramente abaixo do valor estipulado inicialmente, atingiu-se a resistência média prevista para este programa experimental.

Tabela 4: Resultados de resistência à compressão.

\begin{tabular}{c|c|c}
\hline Fibra & Dosagem / \% em volume & $\mathbf{f}_{\mathbf{c m}} / \mathbf{M P a}$ \\
\hline \multirow{4}{*}{ PP A } & 0,33 & $33,4 \pm 0,3$ \\
\cline { 2 - 3 } & 0,50 & $34,1 \pm 0,7$ \\
\cline { 2 - 3 } & 0,66 & $33,3 \pm 0,1$ \\
\hline \multirow{4}{*}{ PP B } & 0,33 & $34,5 \pm 0,9$ \\
\cline { 2 - 3 } & 0,50 & $33,1 \pm 0,2$ \\
\cline { 2 - 3 } & 0,66 & $35,9 \pm 0,7$ \\
\hline \multirow{3}{*}{ Fibra de aço } & 0,19 & $35,5 \pm 0,2$ \\
\cline { 2 - 3 } & 0,32 & $32,1 \pm 0,5$ \\
\cline { 2 - 3 } & 0,45 & \\
\hline
\end{tabular}

\subsection{Ensaio de flexão}

Para cada corpo-de-prova prismático de 150x150x500mm (altura x largura x comprimento), foi obtida uma curva de carga por abertura de fissura. Para cada conjunto de corpos-de-prova do mesmo traço foi determinada a curva média a partir das curvas individuais. A Figuras 3 (a), (b) e (c) apresentam as curvas individuais para a macrofibra PP A na dosagem de $3,0 \mathrm{~kg} / \mathrm{m}^{3}$ (0,33 em volume), para a macrofibra PP B na dosagem de $4,5 \mathrm{~kg} / \mathrm{m}^{3}\left(0,50 \%\right.$ em volume) e para a fibra de aço na dosagem de $25 \mathrm{~kg} / \mathrm{m}^{3}$ ( $0,32 \%$ em volume), respectivamente. As duas primeiras figuras apresentam apenas três curvas individuais porque foi perdido um corpo-deprova em cada ensaio. Percebe-se que as fibras que possuem maior número de filamentos por unidade de volume apresentam menor dispersão nos resultados, no que se refere ao comportamento pós-fissuração. Este resultado é muito similar aos obtidos em pesquisa anterior sendo que, em todos os casos, o comportamento apresentado nas curvas não apresenta os sinais de instabilidade pós-pico [11]. Na figura 4 estão apresentadas as curvas médias de carga por deslocamento vertical dos concretos reforçados com as três fibras. Verifica-se que as fibras acabam por alterar a resistência residual pós-fissuração, com pouca influência na carga de pico, como era esperado, dado que se está trabalhando abaixo do volume crítico de fibras [1]. 


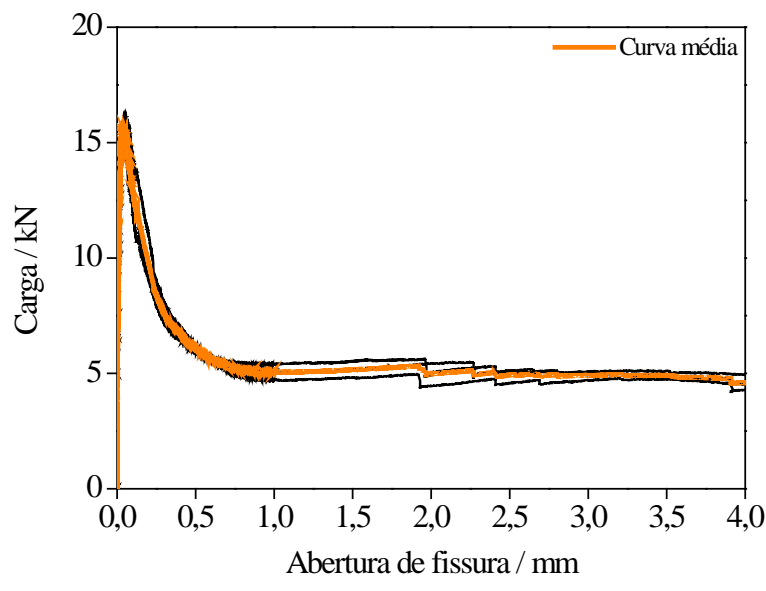

(a)

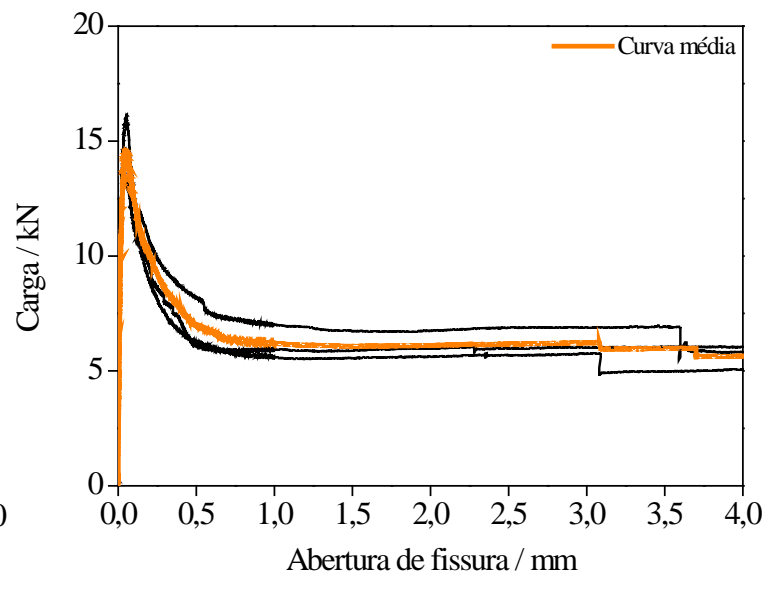

(b)

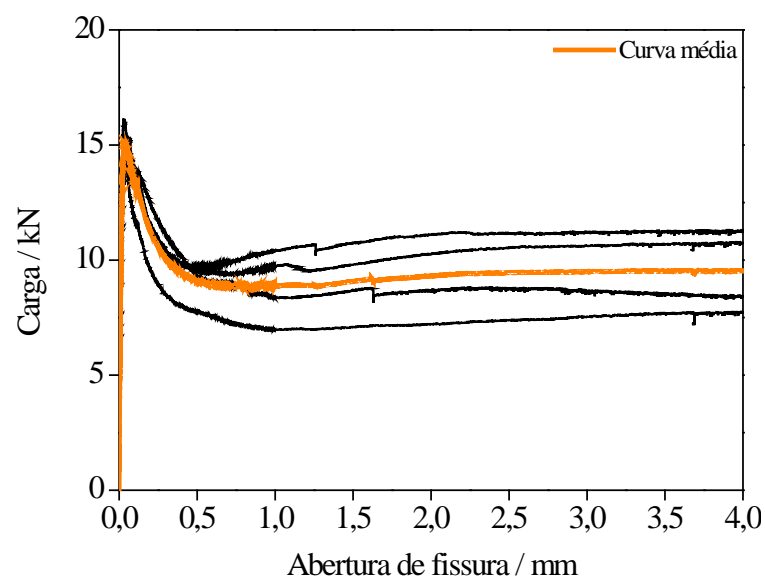

(c)

Figura 3: Curvas individuais e médias obtidas com o concreto reforçado com a macrofibra PP A na dosagem de $3,0 \mathrm{~kg} / \mathrm{m}^{3}$ (a), com a macrofibra PP B na dosagem de $4,5 \mathrm{~kg} / \mathrm{m}^{3}$ (b) e com a fibra de aço na dosagem de $25 \mathrm{~kg} / \mathrm{m}^{3}$ (c).

Em cada gráfico da Figura 4, verifica-se que a resposta elástica inicial, compreendida no intervalo entre 0 e 0,05mm de abertura de fissura, é praticamente inalterada pela adição das fibras, independentemente da dosagem utilizada $[1,13]$. A carga resistida pós-fissuração aumenta com o consumo de fibras e é dependente do módulo de elasticidade da fibra e da sua aderência na matriz. Portanto, a resposta pós-fissuração de concretos reforçados com macrofibras poliméricas ou com fibras de aço tende a ser bastante distinta [25]. Em todos os casos, a ruptura ocorreu com apenas uma fissura discreta (não foi verificada a formação de múltiplas fissuras), o que é típico do baixo volume de fibras utilizado [5] e, naturalmente, da utilização do entalhe no corpo-de-prova [7]. 


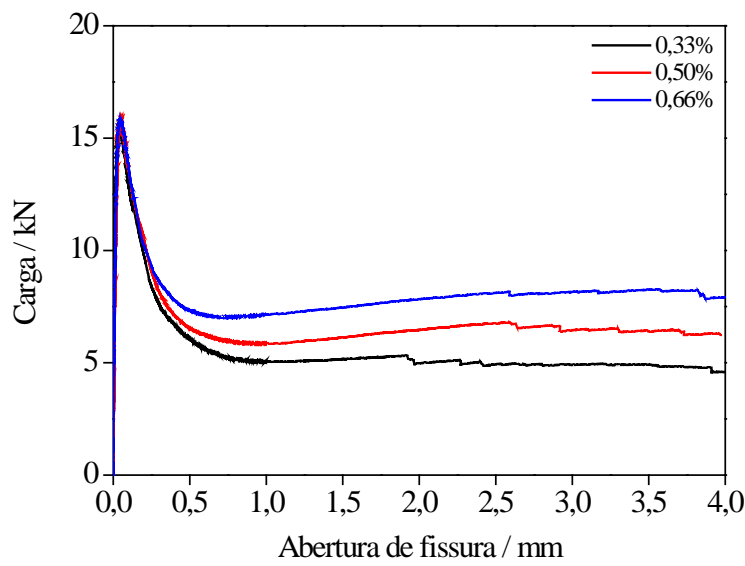

(a)

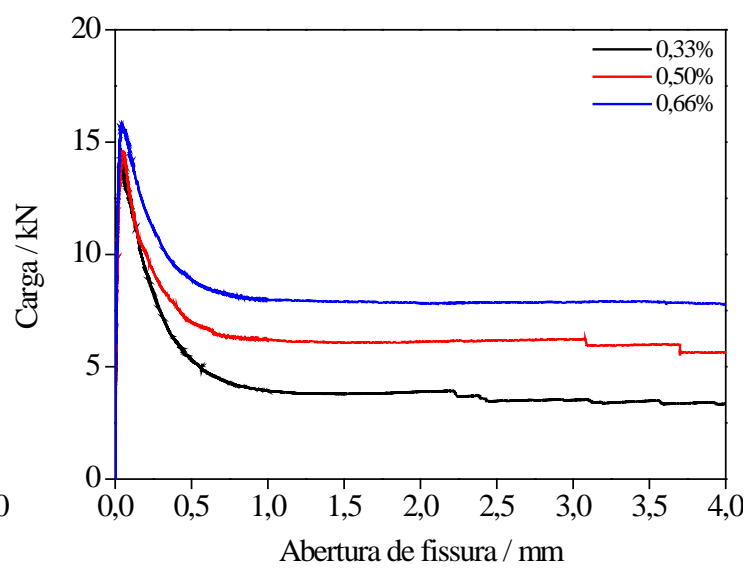

(b)

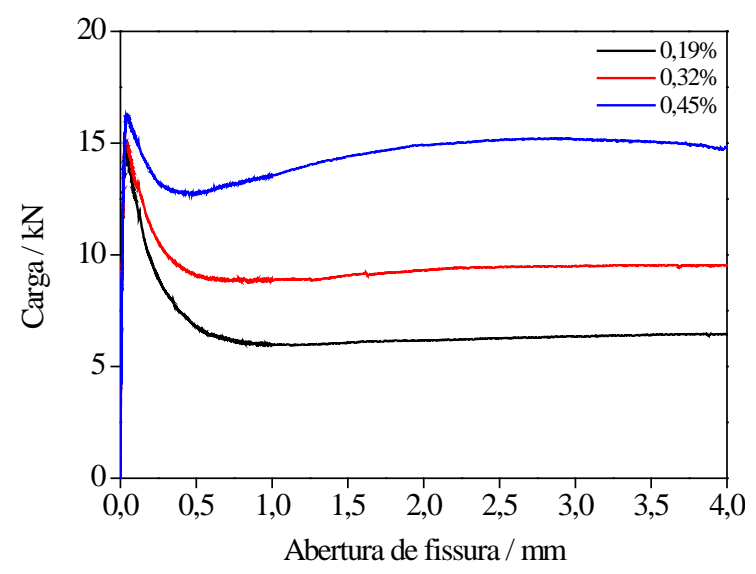

(c)

Figura 4: Curvas médias de carga por abertura de fissura obtidas com o concreto reforçado com a macrofibra PP A (a), com a macrofibra PP B (b) e com a fibra de aço (c).

Como as dosagens das três fibras foram abaixo do volume crítico, o comportamento do compósito após a ruptura da matriz foi de softening. A recuperação da capacidade de absorção de energia é alcançada quando a ancoragem da fibra é mobilizada [5, 29]. Como a fibra de aço apresenta maior rigidez, o ganho de resistência residual é mais intenso para os menores níveis de deformação [11, 31].

Para a macrofibra polimérica, a queda inicial na absorção de energia é mais pronunciada e ocorre em um intervalo de abertura de fissura maior, devido à menor rigidez das fibras poliméricas. Como o rompimento da matriz ocorre com pequenas deformações, as fibras poliméricas têm menor capacidade de absorção de energia imediatamente após a ruptura, quando comparadas às fibras de aço [1]. Entretanto, para grandes aberturas de fissuras, o comportamento tende a ser o oposto, ou seja, a energia absorvida pelas fibras poliméricas pode superar a absorvida pelas fibras de aço [29]. Estes resultados são muito similares aos obtidos em estudos anteriores utilizando distintos métodos de ensaio para o CRF reforçado com fibras de aço e macrofibras poliméricas [11]. Isto demonstra que o comportamento básico do CRF pode ser medido por distintos métodos e o método EN 14651 não apresenta resultados muito diferenciados em relação a outros mais simples.

A partir da análise das curvas experimentais, foram calculadas as resistências residuais em 0,50 e 2,5mm de abertura de fissura, além das relações $f_{R, 1} / L O P$ e $f_{R, 3} / f_{R, 1}$. A partir dos valores médios e desvio padrão foram obtidos os valores característicos (equação 5) e os coeficientes de variação. Os resultados estão apresentados na Tabela 5.

De acordo com os dados apresentados na Tabela 5, pode ser observado que a macrofibra PP A apresentou o comportamento de slip-hardening para os teores de 0,50 e $0,66 \%$ em volume. Isso significa que a resistência residual em $2,5 \mathrm{~mm}$, considerada como a resistência do compósito que pode ser associada ao estado limite último da estrutura [5], é superior à resistência residual em 0,50mm. Tal comportamento também 
foi verificado para a fibra de aço nas dosagens de 0,32 e 0,45\% em volume. Entretanto, a macrofibra PP B apresentou comportamento oposto, denominado de slip-softening, pois a resistência residual em $2,5 \mathrm{~mm}$ é menor do que em $0,50 \mathrm{~mm}$.

Como esperado, a fibra de aço proporciona maior resistência residual do que a macrofibra polimérica para teores equivalentes em volume. Essa diferença é mais acentuada para a resistência na menor abertura de fissura $\left(\mathrm{f}_{\mathrm{R}, 1}\right)$ devido ao alto módulo de elasticidade da fibra de aço.

Tabela 5: Valores de médias e desvio padrão em conjunto com os coeficientes de variação e os valores característicos do limite de proporcionalidade, resistências residuais em 0,50 e 2,50mm de abertura de fissura.

\begin{tabular}{|c|c|c|c|c|c|c|c|c|c|c|}
\hline Fibra & $\begin{array}{c}\text { Dosagem / \% } \\
\text { em volume } \\
\end{array}$ & $\begin{array}{l}\text { LOP / } \\
\text { MPa }\end{array}$ & $\begin{array}{l}\text { CV } \\
(\%) \\
\end{array}$ & $\begin{array}{l}\mathbf{f}_{\mathrm{Lk}} / \\
\mathrm{MPa} \\
\end{array}$ & $\mathbf{f}_{\mathrm{R}, \mathbf{1}} / \mathbf{M P a}$ & $\begin{array}{l}\text { CV } \\
(\%) \\
\end{array}$ & $\begin{array}{l}\mathbf{f}_{\mathrm{R}, \mathbf{1 k}} / \\
\mathbf{M P a}\end{array}$ & $\mathbf{f}_{\mathrm{R}, 3} / \mathbf{M P a}$ & CV (\%) & $\begin{array}{l}\mathbf{f}_{\mathrm{R}, 3 \mathbf{k}} / \\
\mathbf{M P a} \\
\end{array}$ \\
\hline \multirow{3}{*}{ PP A } & 0,33 & $4,46 \pm 0,12$ & 2,69 & 4,26 & $1,72 \pm 0,04$ & 2,33 & 1,65 & $1,41 \pm 0,08$ & 1,28 & 5,67 \\
\hline & 0,50 & $4,70 \pm 0,23$ & 4,89 & 4,32 & $1,86 \pm 0,32$ & 17,20 & 1,33 & $1,94 \pm 0,19$ & 1,63 & 9,79 \\
\hline & 0,66 & $4,56 \pm 0,25$ & 5,48 & 4,15 & $2,08 \pm 0,14$ & 6,73 & 1,85 & $2,26 \pm 0,28$ & 1,80 & 12,39 \\
\hline \multirow{3}{*}{ PP B } & 0,33 & $4,31 \pm 0,27$ & 6,26 & 3,86 & $1,52 \pm 0,09$ & 5,92 & 1,37 & $0,99 \pm 0,10$ & 0,83 & 10,10 \\
\hline & 0,50 & $4,21 \pm 0,34$ & 8,08 & 3,65 & $1,94 \pm 0,32$ & 16,49 & 1,41 & $1,75 \pm 0,17$ & 1,47 & 9,71 \\
\hline & 0,66 & $4,57 \pm 0,26$ & 5,69 & 4,14 & $2,52 \pm 0,20$ & 7,94 & 2,19 & $2,24 \pm 0,06$ & 2,14 & 2,68 \\
\hline \multirow{3}{*}{$\begin{array}{c}\text { Fibra de } \\
\text { aço }\end{array}$} & 0,19 & $4,20 \pm 0,24$ & 5,71 & 3,80 & $1,92 \pm 0,36$ & 18,75 & 1,33 & $1,78 \pm 0,41$ & 1,10 & 23,03 \\
\hline & 0,32 & $4,39 \pm 0,15$ & 3,42 & 4,14 & $2,58 \pm 0,25$ & 9,69 & 2,17 & $2,69 \pm 0,49$ & 1,88 & 18,22 \\
\hline & 0,45 & $4,40 \pm 0,50$ & 11,36 & 3,58 & $3,60 \pm 0,37$ & 10,28 & 2,99 & $4,28 \pm 0,47$ & 3,50 & 10,98 \\
\hline
\end{tabular}

A partir dos resultados apresentados na Tabela 5 foram calculadas as relações de resistências características (equações 3 e 4), apresentadas na Tabela 6. Observa-se que a relação expressa na equação 3 não é atendida para os menores teores de macrofibras poliméricas e para o menor teor de fibra de aço. Ou seja, esse requisito é atendido apenas para os maiores teores de macrofibras de PP e para os teores de fibra de aço acima de $0,32 \%$ em volume. Nota-se também que há uma influência da variabilidade da resistência residual. Isso significa que os teores que apresentaram maior coeficiente de variação na resistência residual $f_{R, 1}(0,5 \%$ para as fibras PP A e PP B, e 0,19\% para a fibra de aço) corresponderam aos menores valores de relação $\mathrm{f}_{\mathrm{R}, 1} /$ $f_{R L}$. Por outro lado, todas as fibras atendem os critérios apresentados na equação 4 , com relações $f_{R, 3} / f_{R, 1}$ superiores ao mínimo exigido de 0,5 . Isto ocorreu pelo comportamento típico de slip-hardening que propiciou valores superiores à unidade para a relação $\mathrm{f}_{\mathrm{R}, 3} / \mathrm{f}_{\mathrm{R}, 1}$ ou mesmo um slip-softening muito pouco pronunciado. Estes critérios definem comportamentos básicos do CRF que irão gerar distintas equações constitutivas que poderão ser utilizadas em projeto [4-6], o que pode ser uma referência muito importante para os projetistas, especialmente os brasileiros. Com isto, se pode obter um maior embasamento técnico da aplicação estrutural do CRF, evitando-se a adoção de teores empíricos em especificações prescritivas sem definição de desempenho mecânico mínimo do material [22]. Este estudo demonstra que a utilização do ensaio da EN 14651 é viável e poderá proporcionar excelente instrumento para o estudo de dosagem e viabilidade do CRF. No entanto, a sua grande complexidade e exigência de cuidado em termos de procedimento experimental podem dificultar a sua utilização para o controle regular onde se poderia aplicar métodos simplificados como os ensaios de sistema aberto ou Barcelona [35].

Por outro lado, há recomendações de utilização de valores médios para a caracterização do concreto reforçado com fibras quando este é destinado a estruturas com alto grau de hiperestaticidade e capacidade de redistribuição de esforços [5]. Nessa condição, segundo critérios de desempenho apresentados no Model Code fib [6], os CRFs utilizados nesse estudo podem receber a classificação mostrada na Tabela 7 e, dessa maneira, todos eles seriam considerados como em condições de serem aplicados com função estrutural, porém com níveis de desempenho distintos obviamente. Vale ressaltar que, se fossem considerados os valores característicos de resistência pós-fissuração, haveria uma redução da classe resistente considerada para sete dos nove traços analisados (Tabela 7). 
Tabela 6: Relações de resistência característica $f_{R, 1 k} / f_{L k}$ e $f_{R, 3 k} / f_{R, 1 k}$ obtidos para traços analisados no experimento segundo o previsto no Model Code fib, 2010.

\begin{tabular}{|c|c|c|c|}
\hline Fibra & Dosagem / \% em volume & Relação $\mathbf{f}_{\mathrm{RLk}} / \mathbf{f}_{\mathrm{Lk}}$ & Relação $\mathbf{f}_{R, 3 k} / \mathbf{f}_{R, 1 k}$ \\
\hline \multirow{3}{*}{ PP A } & 0,33 & 0,39 & 0,77 \\
\hline & 0,50 & 0,31 & 1,22 \\
\hline & 0,66 & 0,45 & 0,97 \\
\hline \multirow{3}{*}{ PP B } & 0,33 & 0,35 & 0,60 \\
\hline & 0,50 & 0,39 & 1,04 \\
\hline & 0,66 & 0,53 & 0,98 \\
\hline \multirow{3}{*}{ Fibra de aço } & 0,19 & 0,35 & 0,83 \\
\hline & 0,32 & 0,52 & 0,87 \\
\hline & 0,45 & 0,84 & 1,17 \\
\hline
\end{tabular}

Os resultados apresentados na Tabela 5 foram utilizados para a produção de gráficos de correlação entre a medida da resistência residual média e o teor de fibra. Foram obtidos os gráficos de dosagem das fibras, apresentados na Figura 5, que permitem determinar o teor de fibra que corresponde à demanda de desempenho média avaliada pelo ensaio de flexão em prismas. A linha de tendência adotada em todos os casos foi a de potência. Alguns estudos anteriores utilizaram outros modelos com eficácia equivalente [32, 33]. Nos dois gráficos da Figura 5, foi obtida excelente aderência da curva de tendência aos dados experimentais, visto que o valor de $\mathrm{R}^{2}$ foi superior a 0,95 em todas as regressões. Como esperado, quando se compara um mesmo teor em volume de fibras de aço e poliméricas, as fibras de aço conferem maiores resistências residuais, principalmente para baixos níveis de deformação, devido à sua maior rigidez. Assim, é possível quantificar o teor de fibra em função do requisito estabelecido para o material. Como exemplo, se for estabelecido um valor de $\mathrm{f}_{\mathrm{R}, 1}$ mínimo de $2 \mathrm{MPa}$, o teor de fibra de aço em volume correspondente seria de $0,21 \%\left(16,5 \mathrm{~kg} / \mathrm{m}^{3}\right)$. Por outro lado, seriam necessários os teores em volume de $0,5 \%\left(4,6 \mathrm{~kg} / \mathrm{m}^{3}\right)$ e $0,6 \%\left(5,5 \mathrm{~kg} / \mathrm{m}^{3}\right)$ para as macrofibras PP B e PP A, respectivamente. Ou seja, o teor em volume das macrofibras poliméricas deveria ser superior ao dobro do teor de fibra de aço para o mesmo nível de desempenho de resistência residual. Este resultado foi muito similar ao obtido em estudo anterior [34].

Tabela 7: Classificação de desempenho segundo critérios apresentados no Model Code fib (2010).

\begin{tabular}{c|c|c|c}
\hline \multirow{2}{*}{ Fibra } & $\begin{array}{c}\text { Dosagem / \% em } \\
\text { volume }\end{array}$ & $\begin{array}{c}\text { Classificação de desempenho } \\
\text { (valor característico) }\end{array}$ & $\begin{array}{c}\text { Classificação de desempenho } \\
\text { (valor médio) }\end{array}$ \\
\hline \multirow{3}{*}{ PP A } & 0,33 & $1,5 \mathrm{a}$ & $1,5 \mathrm{~b}$ \\
\cline { 2 - 4 } & 0,50 & $1,0 \mathrm{~d}$ & $1,5 \mathrm{~b}$ \\
\cline { 2 - 4 } & 0,66 & $1,5 \mathrm{c}$ & $2,0 \mathrm{~b}$ \\
\hline \multirow{3}{*}{ PP B } & 0,33 & $1,0 \mathrm{a}$ & $1,5 \mathrm{a}$ \\
\cline { 2 - 4 } & 0,50 & $1,0 \mathrm{c}$ & $2,0 \mathrm{~b}$ \\
\hline \multirow{3}{*}{ Fibra de aço } & 0,66 & $2,0 \mathrm{c}$ & $2,5 \mathrm{~b}$ \\
\cline { 2 - 4 } & 0,19 & $1,0 \mathrm{~b}$ & $1,5 \mathrm{~b}$ \\
\cline { 2 - 4 } & 0,32 & $2,0 \mathrm{~b}$ & $2,5 \mathrm{~b}$ \\
\hline
\end{tabular}




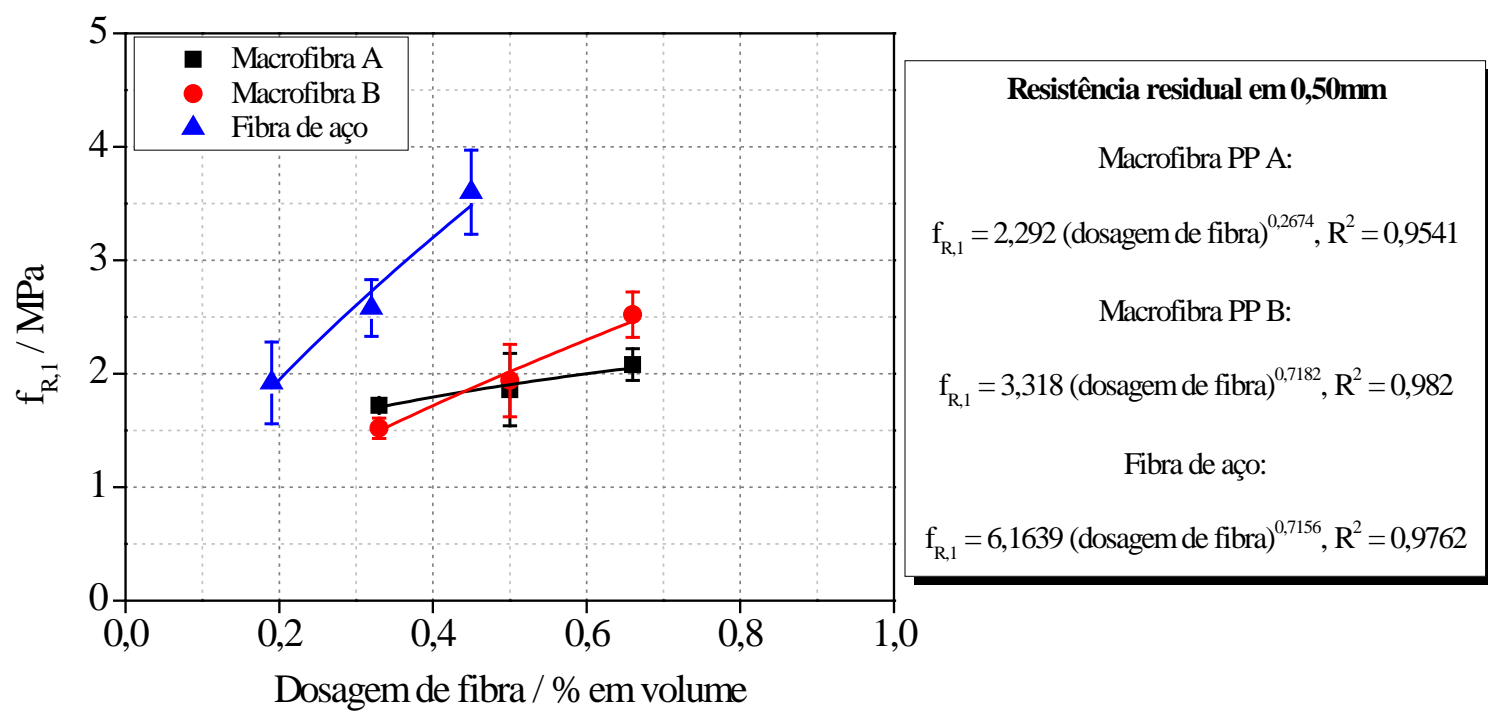

(a)

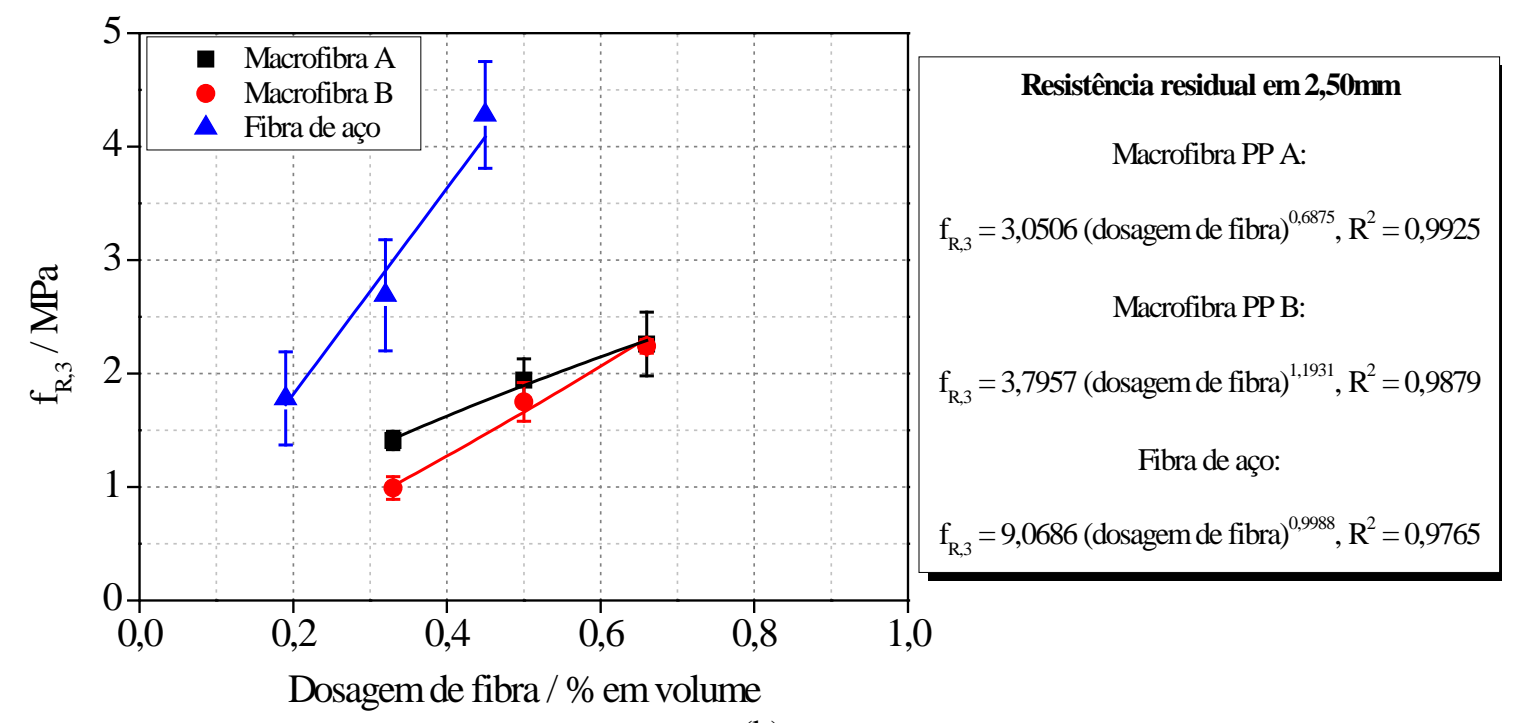

(b)

Figura 5: Correlação entre a resistência residual e o teor de fibras: (a) resistência residual em 0,50mm; (b) resistência residual em 2,5mm.

\section{CONCLUSÕES}

Este trabalho experimental investigou o comportamento de concreto reforçado com fibras quando ensaiado à flexão utilizando sistema fechado de controle de velocidade de deslocamento, segundo a norma EN 14651. A partir dos resultados obtidos, os seguintes pontos podem ser concluídos:

a) Os resultados demonstraram ser possível realizar uma avaliação do comportamento mecânico do CRF através do ensaio EN 14651 gerando subsídios para uma dosagem do material de modo a atender às exigências de resistência residual preconizadas pelo fib Model Code para a sua aplicação como material estrutural. Tanto para o concreto reforçado com fibras de aço como para o reforçado com fibras de PP é possível verificar se atendem aos requisitos gerais da especificação, como também requisitos específicos de resistência residual. Foi possível estabelecer correlações entre as resistências residuais e os teores de fibras com elevado nível de aderência aos pontos experimentais, obtendo-se valores de $\mathrm{R}^{2}$ superiores a 0,95. Estas correlações podem ser utilizadas em estudos de dosagem específico permitindo otimizar o consumo de fibra para cada demanda de projeto e evitandose o uso de teores empíricos pré-fixados [1]. Naturalmente, devido à menor resistência e módulo de elasticidade, os teores de macrofibras poliméricas em volume deverão maiores que os de fibra de aço como já demonstrado em trabalhos anteriores [34]; 
b) Apesar do método da EN 14651 permitir realizar uma avaliação dos diferentes tipos de reforço sem a influência negativa da instabilidade pós-pico [11], há que se considerar que é um ensaio sofisticado e restrito, o que dificulta sua implementação para o controle de qualidade corriqueiro no Brasil. No entanto, o mesmo pode ser utilizado para a realização de estudos prévios de qualificação do material para fins de aplicação estrutural, podendo-se utilizar os requisitos do fib Model Code para tal;

c) Foram obtidos coeficientes de variação muito menores do que o esperado para esse tipo de ensaio. Tradicionalmente se espera coeficientes de variação para a tenacidade da ordem de 20\% [36]. A determinação da tenacidade tende a mitigar o efeito da variabilidade do ensaio em relação à determinação da resistência residual, uma vez que esta se foca numa resistência pontual do ensaio para um dado nível de deslocamento e abertura de fissura. No entanto, os valores de coeficiente de variação obtidos neste estudo associados à resistência residual ficaram abaixo dos 20\% na maioria dos casos. Quando isto não aconteceu houve problemas para considerar o concreto adequado para aplicações estruturais, o que só seria possível se fossem considerados valores médios. Isto demonstra a importância da mitigação da variabilidade do ensaio. Vale ressaltar que o grau de dispersão dos resultados foi inversamente proporcional ao teor de fibras, o que está relacionado com o número de fibras presente na seção de ruptura que, sendo elevado, tem sua capacidade de reforço menos sujeita a pequenas variações na quantidade e direcionamento das fibras;

d) Estas observações demonstram que o ensaio EN 14651 é ideal para ser utilizado em estudos de dosagem prévios à execução da obra, uma vez que permitem também verificar se o CRF atende aos requisitos mínimos especificados pelos novos modelos de dimensionamento.

\section{AGRADECIMENTOS}

Os autores agradecem à empresa Construquímica pelo suporte fornecido para a realização dos ensaios.

\section{BIBLIOGRAFIA}

[1] FIGUEIREDO, A.D. “Concreto com fibras”, In: Concreto: ciência e tecnologia. $1^{\text {a }}$ ed., capítulo 37, São Paulo, Instituto Brasileiro do Concreto (IBRACON), 2011.

[2] BARRAGÁN, B. E., Failure and toughness of steel fiber reinforced concrete under tension and shear, Doctoral thesis, Universitat Politécnica de Catalunya, Barcelona, Spain, 2002.

[3] FIGUEIREDO, A. D. “A nova especificação brasileira das fibras de aço para concreto”, In: 50 Congresso Brasileiro do Concreto, São Paulo, Instituto Brasileiro do Concreto - IBRACON, 2008.

[4] ABECE. "Estruturas de concreto reforçado com fibras - Diretrizes para projeto. Texto Preliminar 1”, In: Associação Brasileira de Engenharia e Consultoria Estrutural (ABECE), Novembro, 2013.

[5] PRISCO, M., et al., "Fiber reinforced concrete in the new fib Model Code", In: $3^{\text {rd }}$ fib International Congress, Washington, p. 1-12, 2010.

[6] FIB Model Code for Concrete Structures 2010, International Federation for Structural Concrete, Switzerland, 2013.

[7] EUROPEAN COMMITTEE FOR STANDARDIZATION. EN 14651: Test method for metallic fiberreinforced concrete - Measuring the flexural tensile strength (limit of proportionality (LOP), residual), CEN, London, 15 p., 2007.

[8] AMERICAN SOCIETY FOR TESTING MATERIALS. ASTM C1609: Standard test method for flexural performance of fiber-reinforce concrete (using beam with third-point loading), Philadelphia, 2010.

[9] AMERICAN SOCIETY FOR TESTING MATERIALS. ASTM C1399: Standard test method for obtaining average residual-strength of fiber-reinforced concrete, Philadelphia, 2010.

[10] THE JAPAN SOCIETY OF CIVIL ENGINEERS. "JSCE-SF4: Method of tests for flexural strength and flexural toughness of steel fiber reinforced concrete”, Concrete library of JSCE, Part III-2 Method of tests for steel fiber reinforced concrete, n. 3, pp. 58-61, 1984.

[11] SALVADOR, R. P., FIGUEIREDO, A. D. “Análise comparativa de comportamento mecânico de concreto reforçado com macrofibra polimérica e com fibra de aço”, Matéria, v.18, n.2, p.1273 - 1285, 2013.

[12] TORRIJOS, M. C., BARRAGÁN, B. E., ZERBINO, R. L. "Placing conditions, mesostructural characteristics and post-cracking response of fiber reinforced self-compacting concretes”, Construction and Building Materials, v. 24, n.6, pp. 1078-1085, 2010.

[13] BURATTI, N., MAZZOTTI, C., SAVOIA, M. "Post-cracking behaviour of steel and macro-synthetic fibre-reinforced concretes”, Construction and Building Materials, v. 25, n.5, pp. 2713-2722, 2011. 
[14] KIM, D. J., PARK, S. H., RYU, G. S., et al.,“Comparative flexural behavior of hybrid ultra high performance fiber reinforced concrete with different macrofibers", Construction and Building Materials, v. 25, n.11, pp. 4144-4155, 2011.

[15] BARROS, J.A.O., CUNHA, V.M.C.F., RIBEIRO, A.F., et al., "Post-cracking behaviour of steel fibre reinforced concrete”, Materials \& Structures (RILEM), v.38, n.1, pp.47-56, 2005.

[16] RAMBO, D.A.S., SILVA, F. A., TOLEDO FILHO, R. D. "Flexural behavior of hybrid steel fiber reinforced self-consolidating concretes”, Revista Escola de Minas (Impresso), v. 67, n.1, pp. 27-32, 2014.

[17] GAVA, G. P., PRUDENCIO JR, L.R., PIERI, T.S. "Techniques to monitor the crack width and the neutral axis position of steel fiber reinforced concrete beams subjected to bending”, Rev. IBRACON Estrut. Mater, v.2, n.1, pp. 59-84, 2009.

[18] OLIVEIRA JUNIOR, L. A., ARAÚJO, D. L., TOLÊDO FILHO, R. D., et al., "Influence of steel fibers and mineral additions on cracking behavior of reinforced concrete tension members", Rev. IBRACON Estrut. Mater, v.2, n.2, pp. 142-165, 2009.

[19] BARROS, A. R., GOMES, P. C. C., BARBOZA, A. S. R. "Steel fibers reinforced self-compacting concrete: behavior to bending”, Rev. IBRACON Estrut. Mater., v.4, n.1, pp. 49-78, 2011.

[20] MIGLIORINI, A., GUIMARÃES, A. T. C., OZORIO, B. M. "Fibras de aço em blocos de concreto: estudo para utilização em ambiente marítimo”, Matéria, v. 16, pp. 997-1008, 2012.

[21] DEBS, M. K. "Emprego de compósitos de cimento reforçados com fibras nas ligações de concreto prémoldado”, Revista do IBRACON - Instituto Brasileiro do Concreto, São Paulo, v. 34, n.43, pp. 80-86, 2006.

[22] FIGUEIREDO, A. D.“Os desafios para o uso do concreto reforçado com fibras como material estrutural “, In: $56^{\circ}$ Congresso Brasileiro do Concreto, Natal, 2014.

[23] GOPALARATNAM, V. S. "On the characterization of flexural toughness in fiber reinforced concretes", Cement \& Concrete Composites, v. 17, n.3, pp. 239-254, 1995.

[24] THE JAPAN SOCIETY OF CIVIL ENGINEERS. "JSCE-SF2: Method of making specimens for strength and toughness tests of steel fiber reinforced concrete”, Concrete library of JSCE. Part III-2 Method of tests for steel fiber reinforced concrete, n. 3, pp. 48-55, 1984.

[25] ASSOCIAÇÃO BRASILEIRA DE NORMAS TÉCNICAS. NBR 5739: Concreto: ensaio de compressão de corpos-de-prova cilíndricos, Rio de Janeiro, 2007, 9p.

[26] CECCATO, M. R., NUNES, N. L., FIGUEIREDO, A. D., "Estudo do controle da trabalhabilidade do concreto reforçado com fibras de aço”, In: IV Congresso Iberoamericano de Patologia das Construções e VI Congresso de Controle de Qualidade CONPAT97, pp. 539-546, Porto Alegre, v. 2, 1997.

[27] NAYAR, S. K., GETTU, R. "On the design of steel fibre reinforced concrete pavements and slabs-ongrade”, In: 8th International RILEM Symposium on Fiber Reinforced Concrete: challenges and opportunities (BEFIB 2012), pp. 1070-1081, Portugal, RILEM Publications S.A.R.L., 2012.

[28] ARMELIN, H. S. Contribuição ao estudo do concreto projetado por via seca com fibras de aço destinado ao revestimento de túneis NATM, Dissertação de M.Sc., Escola politécnica, Universidade de São Paulo, 1992.

[29] CONCRETE SOCIETY. Technical Report $n^{\circ}$ 65, Guidance on the use of macro-synthetic-fiberreinforced concrete, 76p., The Concrete Society, Camberley, 2007.

[30] BANTHIA, N., DUBEY, A., "Measurement of flexural toughness of fiber-reinforced concrete using a novel technique-Part 2: Performance of Various Composites”, ACI Materials Journal, v.97, n.1, pp.3-11, 2000.

[31] ARAKAKI, D. H., FIGUEIREDO, A. D. "Avaliação da tenacidade de concretos reforçados com fibras de aço de alto e baixo teor de carbono”, In: IV Simpósio EPUSP sobre Estruturas de Concreto, São Paulo, 2000.

[32] FIGUEIREDO, A. D., NUNES, N. L., TANESI, J. “Mix design analysis on steel fiber reinforced concrete”, In: Fifth International RILEM Symposium on Fibre-Reinforced Concretes (FRC), Lyon, pp.103 -118, 2000.

[33] ARMELIN, H.S., BANTHIA, N. "Predicting the flexural post-cracking performance of steel fiberreinforced concrete from the pull-out of single fibers”, ACI Materials Journal, Detroit, USA, 1997. 
[34] FIGUEIREDO, A. D., FUENTE, A., AGUADO, A., et al. "Fibras plásticas como reforço de tubos de concreto. Parte 1: Caracterização tecnológica”, In: 54 Congresso Brasileiro do Concreto, Maceió, IBRACOM, 2012.

[35] MONTE, R., TOALDO, G. S., FIGUEIREDO, A. D. “Avaliação da tenacidade de concretos reforçados com fibras através de ensaios com sistema aberto”, Matéria , v.19, pp.132 - 149, 2014.

[36] MORGAN, D.R., MINDESS, S., CHEN, L. "Testing and Specifying Toughness for Fiber Reinforced Concrete and Shotcrete”, In: Second University-Industry Workshop on Fiber Reinforced Concrete and Other Advanced Materials, pp.29-50,Toronto, Canada, 1995. 